\title{
Gram-scale cryogenic calorimeters for rare-event searches
}

\author{
R. Strauss, ${ }^{1,}$ J. Rothe, ${ }^{1}$ G. Angloher, ${ }^{1}$ A. Bento, ${ }^{2}$ A. Gütlein, ${ }^{3}$ D. Hauff, ${ }^{1}$ H. Kluck, ${ }^{3}$ M. Mancuso, ${ }^{1}$ L. Oberauer, ${ }^{4}$ \\ F. Petricca, ${ }^{1}$ F. Pröbst, ${ }^{1}$ J. Schieck, ${ }^{3}$ S. Schönert, ${ }^{4}$ W. Seidel,,${ }^{1, \dagger}$ and L. Stodolsky ${ }^{1}$ \\ ${ }^{1}$ Max-Planck-Institut für Physik, D-80805 München, Germany \\ ${ }^{2}$ CIUC, Departamento de Fisica, Universidade de Coimbra, P3004 516 Coimbra, Portugal \\ ${ }^{3}$ Institut für Hochenergiephysik der Österreichischen Akademie der Wissenschaften, \\ A-1050 Wien, Austria, and Atominstitut, Vienna University of Technology, A-1020 Wien, Austria \\ ${ }^{4}$ Physik-Department, Technische Universität München, D-85748 Garching, Germany
}

(Received 14 April 2017; published 28 July 2017)

\begin{abstract}
The energy threshold of a cryogenic calorimeter can be lowered by reducing its size. This is of importance since the resulting increase in signal rate enables new approaches in rare-event searches, including the detection of $\mathrm{MeV}$ mass dark matter and coherent scattering of reactor or solar neutrinos. A scaling law for energy threshold vs detector size is given. We analyze the possibility of lowering the threshold of a gram-scale cryogenic calorimeter to the few eV regime. A prototype $0.5 \mathrm{~g} \mathrm{Al}_{2} \mathrm{O}_{3}$ device achieved an energy threshold of $E_{\mathrm{th}}=(19.7 \pm 0.9) \mathrm{eV}$, the lowest value reported for a macroscopic calorimeter.
\end{abstract}

DOI: 10.1103/PhysRevD.96.022009

\section{INTRODUCTION}

Cryogenic calorimetry [1] is based on the idea that the temperature rise in a target after an energy deposition $\Delta E$ is given by

$$
\Delta T=\frac{\Delta E}{C}
$$

where $C$ is the heat capacity of the object. A small $C$, which can be achieved in crystalline materials at $\sim \mathrm{mK}$ temperatures, leads to a large temperature jump and so to a high sensitivity to small energies. State-of-the-art cryogenic detectors with a mass of $300 \mathrm{~g}$ have reached energy thresholds down to $\sim 300 \mathrm{eV}$ [2].

A further reduction of the threshold is of great interest since for many important processes, such as coherent neutrino nucleus scattering (CNNS) or the scattering of the hypothetical dark matter (DM) particles, the count rate increases strongly as the threshold of a detector is lowered. In Fig. 1 we show the count rate in a $\mathrm{CaWO}_{4}$ detector for various processes as a function of the recoil energy $E_{R}$ : For CNNS of antineutrinos at a distance of $40 \mathrm{~m}$ from a $4 \mathrm{GW}$ nuclear power reactor (thick full line), for a hypothetical DM particle with a mass of $200 \mathrm{MeV} / \mathrm{c}^{2}$ and a cross section of $1 \mathrm{pb}$ (dashed line), and for CNNS of solar neutrinos (full line). Measured background rates at different shallow low-background facilities [3,4], extrapolated to lower energies, are shown as a grey band. The lower limit (dotted line) indicates the present fundamental background limitation due to the intrinsic radiopurity of $\mathrm{CaWO}_{4}$

\footnotetext{
*Corresponding author

strauss@mpp.mpg.de

Deceased.
}

crystals, as measured deep underground [5]. One observes that a threshold in the $10 \mathrm{eV}$ regime can allow the count rates to rise significantly above background levels. Below, we show that a rapid detection of CNNS at a nuclear reactor is in reach and that the technology offers unique possibilities for the detection of MeV-scale DM and solar neutrinos.

Since macroscopic amounts of target material are necessary to obtain reasonable count rates for such weak interaction processes, microscopic calorimeters with target masses below milligrams are impractical for rare-event searches. Here we describe how it is possible to achieve a

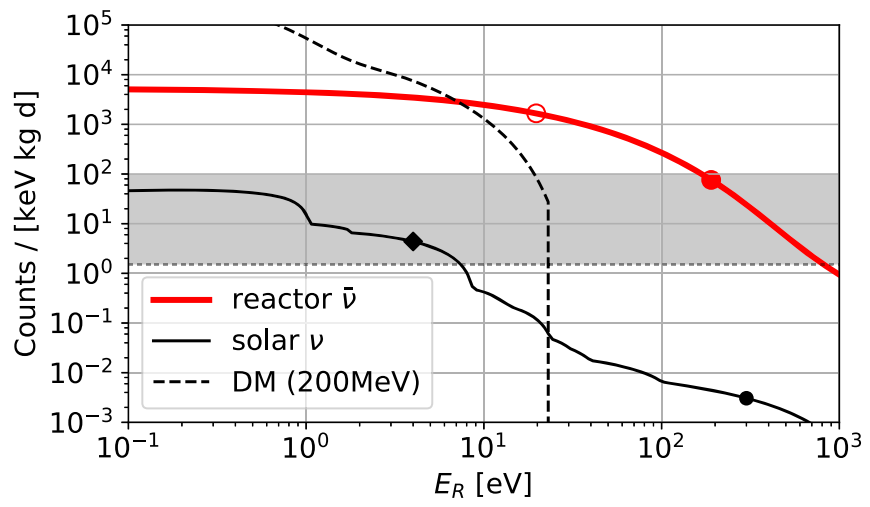

FIG. 1. Nuclear-recoil spectra on $\mathrm{CaWO}_{4}$ for CNNS of antineutrinos from a $4 \mathrm{GW}$ nuclear reactor at a distance of $40 \mathrm{~m}$, for $200 \mathrm{MeV} / \mathrm{c}^{2}$ mass DM $(\sigma=1 \mathrm{pb})$, and for CNNS of solar neutrinos. The grey area indicates a range of measured background levels for shallow sites [3,4] and at a deep-underground location (dotted line) [5]. Closed circles indicate previous cryogenic detector technology [2,6]; the performance of the detector presented here is depicted as an open circle (surface operation). The expected threshold of the presented detector at a deep-underground location is shown as a diamond. 
suitably low threshold with a small but macroscopic calorimeter, and present the results of a $0.5 \mathrm{~g}$ prototype.

\section{GRAM-SCALE CRYOGENIC CALORIMETERS}

A straightforward application of Eq. (1) would seem to suggest that an improved response for small energies is to be achieved by simply reducing $C$ by lowering the temperature or using smaller devices [1].

However, there are some subtleties in applying Eq. (1). For example it may apply not to the whole device in question, but to some sensitive subsystem. This explains why we obtain results with macroscopic devices, on the order of grams in mass, which one would have thought only possible with very small or microscopic systems. Below we report an energy threshold of $\sim 20 \mathrm{eV}$-which is in the order of atomic binding energies-for a $0.5 \mathrm{~g}$ device operated under unshielded conditions.

\section{A. Pulse height}

To understand this apparently surprising result, we consider the operation of the detectors similar to those used in Cryogenic Rare Event Search with Superconducting Thermometers (CRESST) for direct dark matter search [2,7]. These detectors operate out of thermal equilibrium after an energy deposition. The signal originates from the initial ballistic nonthermal phonons of the particle event occurring in the absorber crystal. The deposited energy is measured using a superconducting transition-edge sensor (TES). This is explained at length in Ref. [8], and we use the terminology and notation of this reference. In particular, we deal with detectors operating in the calorimetric mode (Sec. 3.3.1 in [8]), where the film thermalizes slowly with respect to the signal duration and so integrates the energy of the incoming nonthermal phonons. This results in a temperature rise in the thermometer film given by

$$
\Delta T_{\text {film }}=\epsilon \Delta E / C_{e}
$$

where $\epsilon$ is the fraction of the deposited energy $\Delta E$ thermalized in the film, and $C_{e}$ is the heat capacity of the electrons in the film.

Since $\epsilon \Delta E$ is the energy absorbed in the film, Eq. (2) amounts to Eq. (1), but applied to the electrons of the film. Their temperature rise and thus our readout signal is given by a pulse whose magnitude originates in a microscopic system, although the detector itself is macroscopic.

A fundamental limitation only arises through the fact that for very small $C$ a body coupled to a heat bath has large irreducible temperature fluctuations $(\Delta T / T)^{2}=1 / C$ [9], where we use $k_{B}=1$. This corresponds to theoretical energy resolutions of $\mathcal{O}(1 \mathrm{eV})$ for massive calorimeters with masses of $\sim 100 \mathrm{~g}$ [10]. This is not a significant limitation for the detectors we consider $\left(C \sim 10^{8}\right.$ for the $\mathrm{W}$ film used here), but it does set a limit on an indefinite reduction of $C$.

\section{B. Scaling law}

A simple scaling law enables us to extrapolate results with previous CRESST detectors to smaller sizes. This is possible on the basis of Eq. (2). We are interested in the threshold energy $E_{t h}$, which is inversely proportional to the temperature rise $\Delta T_{\text {film }}$ for a given energy deposition.

We arrive at the following approximate scaling law for threshold energy vs detector mass $M$ :

$$
E_{\mathrm{th}}=(\text { const }) \times M^{2 / 3} .
$$

The proportionality constant depends on the material and geometry but not on the size of the crystal. Further, constant noise conditions and a similar performance of the TES sensors is assumed.

Eq. (3) results from Eq. (2) as follows. The appearance of $\epsilon$, representing the fraction of the original nonthermal phonons thermalizing in the film, shows that the main effect entering in Eq. (2) is the competition between the thermalization in the film and the thermalization on the surfaces of the crystal. We expect that $\epsilon$ is small due to the great difference in the respective surface areas and is simply given the ratio of thermalization rates which we write as $\kappa A$. Taking the ratio for the film and crystal surface one has $\epsilon=\frac{\kappa_{\text {film }} A_{\text {film }}}{\kappa_{\text {crystal }} A_{\text {crystal }}}$, where the $\kappa$ refer to material properties and the $A$ to the surface areas. Inserting in Eq. (2) and keeping only factors related to size, one obtains

$$
\epsilon \frac{\Delta E}{C_{e}}=\frac{\kappa_{\text {film }} A_{\text {film }}}{\kappa_{\text {crystal }} A_{\text {crystal }}} \frac{\Delta E}{C_{e}} \propto \frac{1}{A_{\text {crystal }}},
$$

where we have left out all factors that do not depend on the crystal size in the last step. $A_{\text {film }}$ has canceled because $C_{e} \propto V_{\text {film }}=A_{\text {film }} \cdot h$, and the films all have about the same thickness $h$. Eq. (4) shows that the main effect in reducing the size of the crystals is to reduce their surface area and so increase the signal correspondingly. For a cube one has $A_{\text {crystal }} \propto d^{2}$ and $M \propto d^{3}$ where $d$ is the edge length. Since the threshold varies inversely to the pulse height or $\Delta T_{\text {film, }}$, we obtain Eq. (3).

Figure 2 shows the scaling law for $\mathrm{CaWO}_{4}$ (dashed line) and $\mathrm{Al}_{2} \mathrm{O}_{3}$ (dotted line) calorimeters vs mass operated in the CRESST low-background setup, here referred to as the benchmark setup. The offset between the dashed and dotted lines is due to the different density and sound speed of the materials, and to material-dependent transmission properties of phonons into the film (total factor $\sim 0.77$ ). The model is fitted to the results of $\mathrm{CaWO}_{4}$ detectors with $M=250-300 \mathrm{~g}$ (triangles) used in CRESST-II $[2,11]$ and a $\mathrm{Al}_{2} \mathrm{O}_{3}$ cube of $M=262 \mathrm{~g}$ (cross) used in CRESST-I [12]. The scaling law nicely matches the performance of 


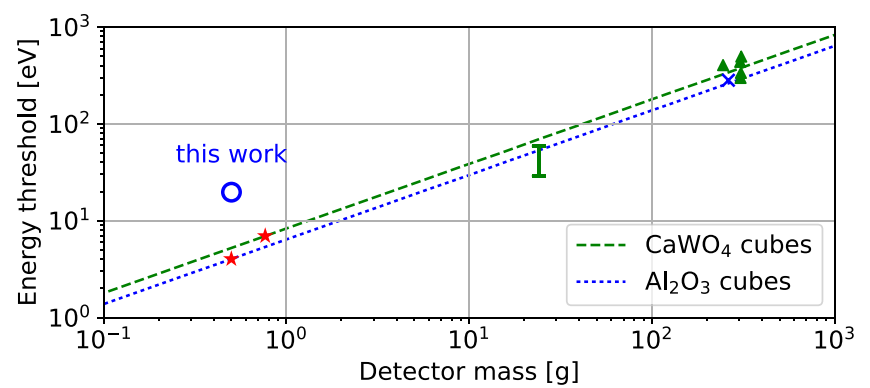

FIG. 2. The scaling law for $\mathrm{CaWO}_{4}$ and $\mathrm{Al}_{2} \mathrm{O}_{3}$ cubes fitted to results of cryogenic $\mathrm{CaWO}_{4}$ (triangles) and $\mathrm{Al}_{2} \mathrm{O}_{3}$ (cross) detectors $[2,11,12]$. The lines show the $M^{2 / 3}$ behavior according to Eq. (3). The expected values for the prototype described here under low-background conditions are shown by the red stars. There is agreement with the expected performance of $24 \mathrm{~g}$ $\mathrm{CaWO}_{4}$ detectors (green error bar) [6]. The result presented here is shown by a blue circle.

$24 \mathrm{~g} \mathrm{CaWO}_{4}$ detectors for CRESST-III as inferred from an above-ground measurement [6]. The error bar indicates the threshold range of this device when operated in the benchmark setup depending on the noise level of the individual readout channel [6]. Red stars show the projected performance of $(5 \times 5 \times 5) \mathrm{mm}^{3} \mathrm{CaWO}_{4}$ and $\mathrm{Al}_{2} \mathrm{O}_{3}$ calorimeters. Below we discuss the result of a prototype $0.5 \mathrm{~g} \mathrm{Al}_{2} \mathrm{O}_{3}$ detector operated in a surface test setup (blue circle).

\section{RESULTS FROM A PROTOTYPE}

For the first test of a gram-scale calorimeter, an $\mathrm{Al}_{2} \mathrm{O}_{3}$ cube of $(5 \times 5 \times 5) \mathrm{mm}^{3}$ with a mass of $0.5 \mathrm{~g}$ was used. All surfaces were optically polished. The cube was equipped with a W thin film TES similar to those used for CRESST light detectors [13]. The TES design was adjusted for operation in the "calorimetric mode" [8] on the cubic crystal.

The data presented here were acquired in a cryostat at the Max-Planck-Institut for Physics in Munich, Germany. The setup at surface level had no shielding against environmental radioactivity or cosmogenic backgrounds. The crystal was placed on three $\mathrm{Al}_{2} \mathrm{O}_{3}$ spheres with a diameter of $1 \mathrm{~mm}$ resting on a copper plate and providing pointlike contacts. From the top, a bronze clamp (with a central $\mathrm{Al}_{2} \mathrm{O}_{3}$ sphere) presses on the cube. The electrical and thermal connections are realized by $\mathrm{Al}$ and $\mathrm{Au}$ wire bonds (diameter $25 \mu \mathrm{m}$ ), respectively. The cryostat reached a base temperature of $6 \mathrm{mK}$ and the mixing chamber was stabilized during the measurement at $11 \mathrm{mK}$. The $\mathrm{W}$ TES on the $\mathrm{Al}_{2} \mathrm{O}_{3}$ crystal showed a normal-to-superconducting transition at $22 \mathrm{mK}$. Commercial SQUID magnetometers and a state-of-the-art data acquisition system were used [7].

$\mathrm{A}^{55} \mathrm{Fe}$ x-ray calibration source with an activity of $0.6 \mathrm{~Bq}$ was installed about $2 \mathrm{~cm}$ from the $\mathrm{Al}_{2} \mathrm{O}_{3}$ cube. The detector was neither shielded against external radiation nor against radioactivity originating from materials and surfaces inside the experimental volume. The data amounted to a total measuring time of $5.3 \mathrm{~h}$ corresponding to an exposure of $0.11 \mathrm{~g}$ days. The total particle pulse rate was $0.36 \mathrm{~Hz}$ of which $\gtrsim 40 \%$ was from the $\mathrm{x}$-ray lines of the ${ }^{55} \mathrm{Fe}$ calibration source.

The particle pulses can be well described by the thermal model for cryogenic detectors [8]. A fit of the model to the template pulse confirms that the $\mathrm{Al}_{2} \mathrm{O}_{3}$ detector is operating in the calorimetric mode. The rise time, which corresponds to the lifetime of the nonthermal phonons, is $\tau_{n}=(0.30 \pm 0.01) \mathrm{ms}$. The two decay times of the model, which depend on the thermal couplings of the TES and the absorber crystal to the thermal bath, are found to be $\tau_{\text {fast }}=(3.64 \pm 0.01) \mathrm{ms}$ and $\tau_{\text {slow }}=(28.17 \pm 0.09) \mathrm{ms}$.

\section{A. Energy calibration}

The energy of an event is inferred from its pulse height. The pulses were fitted by a template pulse extracted from an energy region were the pulse response is completely linear, in this case around $\sim 0.32 \mathrm{~V}$, which corresponds to an energy of about $0.47 \mathrm{keV}$. The detector response becomes increasingly nonlinear at about $3 \mathrm{keV}$. To reconstruct the energy of large, saturated, pulses the method of a truncated template fit was used, a standard method in cryogenic calorimetry (see e.g. [13]). The pulse shape is fitted in the linear region up to the truncation limit, which here is chosen at $0.4 \mathrm{~V}$. Figure 3 (upper frame) shows a $100 \mathrm{eV}$ pulse which is nicely fitted by the template pulse (red line). In comparison, a $5.9 \mathrm{keV}$ pulse from the ${ }^{55} \mathrm{Fe}$ source (lower frame) is depicted. The event is only fit up to the truncation limit. Above this limit the pulse shapes deviate from that of the template. Figure 4 (main frame) shows the fit goodness (rms value) of the truncated template fit (black) in comparison to a template fit without truncation (red). The truncated fit shows no significant

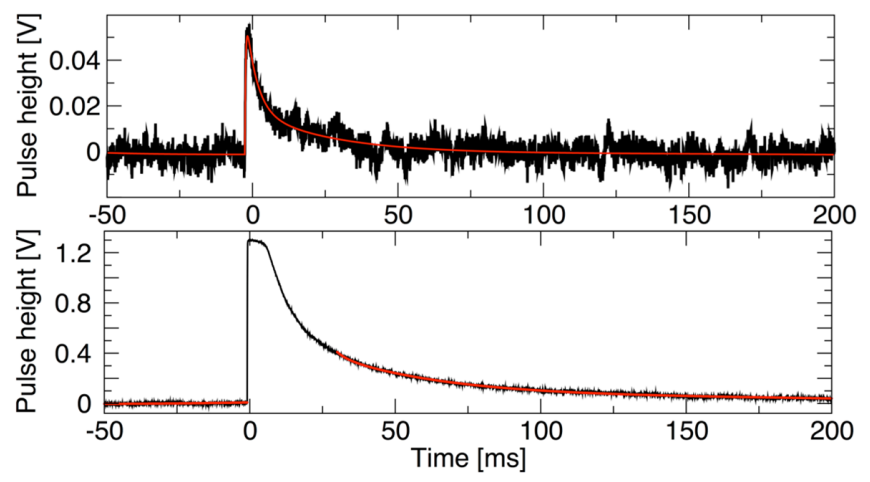

FIG. 3. Randomly chosen pulses from different energy ranges fitted with the truncated template (red lines). Upper frame: An event with an energy of about $100 \mathrm{eV}$ where the detector response is entirely linear. Lower frame: A saturated $5.9 \mathrm{keV}$ pulse. Above $0.4 \mathrm{~V}$ the pulse shapes deviate significantly from the template pulse. 


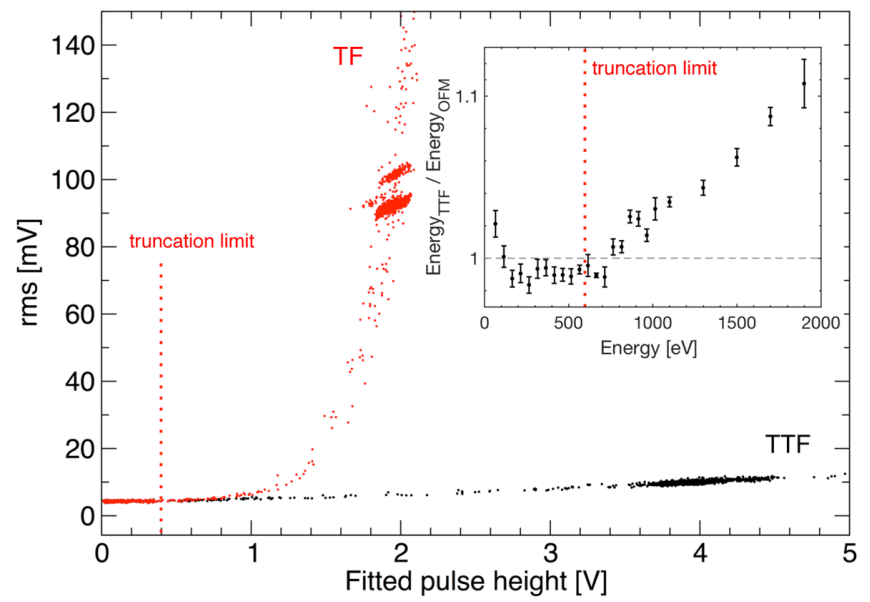

FIG. 4. Main frame: Goodness of the template fit (TF; rms value). The truncation level is $0.4 \mathrm{~V}$ and the truncated template fit (TTF) yields an almost energy-independent rms value (black). The ${ }^{55} \mathrm{Fe} \mathrm{K}_{\alpha}$ x-ray line is reconstructed at a pulse height of $(3.925 \pm 0.003) \mathrm{V}$. The impact of pulse saturation on the energy reconstruction is demonstrated by a classic TF without truncation (red). The rms value rises significantly for energies above the linear region. Inset: Ratio of the reconstructed energy by the TTF and the optimum filter method (OFM). In the linear region both methods agree on the $1 \%$ level (see the text). Above the truncation limit the OFM fails to reconstruct the correct energy, as expected, due to a different pulse shape caused by saturation.

energy dependence of the rms values over the considered energy range. The slight rise (factor of $\sim 2$ ) is expected since only part of the recorded pulse samples is exploited for the energy reconstruction. Without truncation, the fit fails above the linear region since the pulse shape of the events deviates significantly from the template. The dominant $\mathrm{K}_{\alpha}$ line $\left(E_{\text {lit }}=5.895 \mathrm{keV}\right)$ is found to have a pulse height of $(3.925 \pm 0.003) \mathrm{V}$ and is used for the calibration of the pulse spectrum. The energy of the $K_{\beta}$ line on the right shoulder is then found at an energy of $E_{K_{\beta}}=(6.485 \pm 0.017) \mathrm{keV}$, which is in good agreement with the literature value of $6.490 \mathrm{keV}$.

The energy reconstruction of the calibration line is robust against a change of the truncation limit. A variation of $20 \%$ corresponds to a moderate error of $1.1 \%$ on the energy calibration. This value is considered as a systematic error for the energy threshold (see below). Even a dramatic variation of the truncation limit by a factor of 2 changes the reconstructed pulse height of the $\mathrm{x}$-ray lines by only $\sim 3 \%$.

\section{B. Energy spectrum}

Figure 5 shows the final energy spectrum up to $10 \mathrm{keV}$ after stability and (standard) data-quality cuts (see e.g. $[2,11])$. The energy resolution of the reconstructed $\mathrm{x}$-ray lines is $\sigma_{\mathrm{Fe}}=(0.147 \pm 0.005) \mathrm{keV}$, which is significantly larger than the baseline resolution at zero energy, as determined from the variance of the baseline (see below).

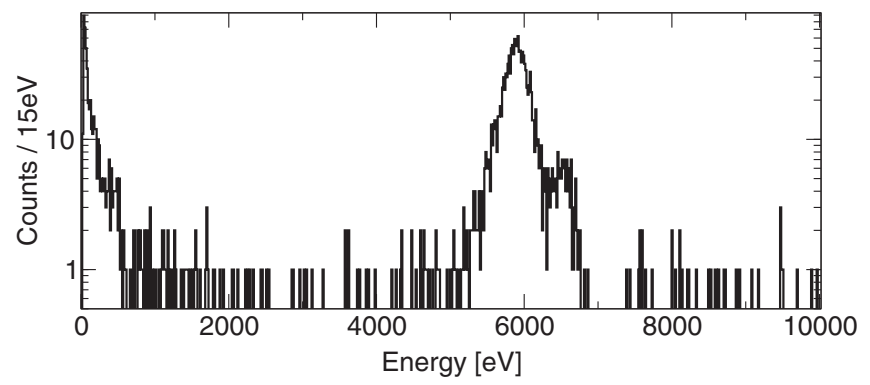

FIG. 5. Final energy spectrum up to $10 \mathrm{keV}$ acquired with the prototype $\mathrm{Al}_{2} \mathrm{O}_{3}$ detector in the presence of a ${ }^{55} \mathrm{Fe}$ calibration source.

Part of this degradation (41\%) is due to the truncated fit that uses only partial information of pulses at higher energies $(\gtrsim 600 \mathrm{eV}$ ), as shown in Fig. 3 (bottom). The remaining energy dependence of the detector resolution (by a factor of 23 at $5.9 \mathrm{keV}$ ) was observed previously for cryogenic $\mathrm{Al}_{2} \mathrm{O}_{3}$ detectors and matches the results of [14].

The measurement shows a constant background rate of $\sim 1.2 \times 10^{5}$ counts/(kg keV day) (7-10 keV) above the calibration peaks; see Fig. 5. This background rate is not unexpected due to the lack of any shielding against ambient and cosmogenic backgrounds. At lower energies $(\lesssim 1 \mathrm{keV})$ the event rate significantly rises towards threshold. Auger electrons from the ${ }^{55} \mathrm{Fe}$ source and surface-contamination induced backgrounds are the most plausible explanation for this increase.

Earlier results of cryogenic detectors operated in lowbackground setups, e.g. [2], show a flat background on a level of 10 counts/(kg keV day) (4-5 orders of magnitude lower compared to this measurement) down to the threshold energy of $300 \mathrm{eV}$. This clearly demonstrates that by a proper selection of the materials surrounding the detector, the surface background contribution can be drastically reduced. An active veto system by using Si slabs equipped with TESs which surround the calorimeter is planned. A dedicated Monte Carlo study shows that surface backgrounds can be reduced to negligible values [15].

\section{Threshold determination}

For the pulse height evaluation at low energies, the optimum filter method is used (see e.g. [16,17]). The optimum filter maximizes the signal to noise for a known signal, in our case the template pulse, in the presence of stochastic noise with a known power spectrum. To build the filter transfer function, the Fourier transform of the template pulse and the noise power spectrum are required. Here, the latter is derived from $\sim 400$ randomly chosen baseline samples. In the selection of the baseline samples the same quality cuts were applied as for the pulse samples. In the frequency domain, the optimum filter weights the spectral components according to their signal-to-noise ratio. Usually the filter is applied in frequency space to 

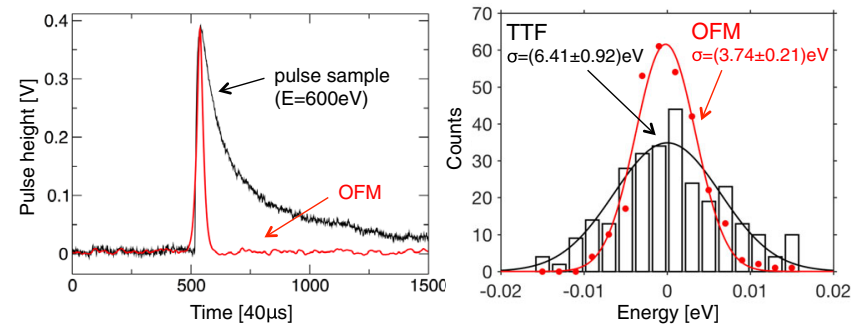

FIG. 6. Left: Pulse sample and optimum filter output in the time domain. Right: Comparison of the baseline noise derived by the TTF (black histogram) and the OFM (red dots). Gaussian fits to the data are shown.

minimize the computing time and is then transformed back to the time domain. The result is normalized so that it reproduces the unfiltered pulse height at the pulse's maximum (see Fig. 6, left). The energy reconstruction by the optimum filter agrees with that of the truncated template fit on a $1 \%$ level in the linear region (up to $600 \mathrm{eV}$ ) and deviates significantly above as expected due to a different pulse shape caused by saturation (see Fig. 4, inset). Below the truncation limit, a maximal deviation of $2.8 \%$ is observed which is considered as systematic error of the energy calibration. The baseline energy resolution after filtering is found to be $\sigma_{b}=(3.74 \pm 0.21) \mathrm{eV}$. This compares to a value of $(6.42 \pm 0.92) \mathrm{eV}$ without filtering, showing a clear improvement (see Fig. 6, right). Accordingly, this effect reduces the energy threshold. This improvement can be exploited using a data acquisition system which continuously streams the detector output, so that the pulse triggering can be done in postprocessing, when signal and noise power spectra are known.

The functionality of such a software trigger based on the optimum filter is illustrated in Fig. 7. A small artificial pulse is superimposed on a randomly selected baseline sample, drawn in the upper frame. The lower frame shows

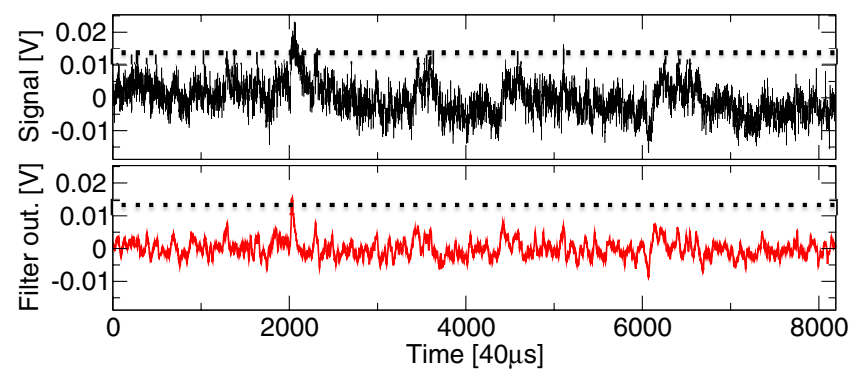

FIG. 7. Demonstration of the optimum trigger. Upper plot: A $19.7 \mathrm{eV}$ standard pulse is superimposed on a randomly chosen noise sample (onset at sample 2000). Lower plot: Output of the optimum filter applied to the sample. The pulse is clearly triggered while noise contributions are suppressed sufficiently below threshold, which is set at a pulse height of $13.0 \mathrm{mV}$ (see the text).

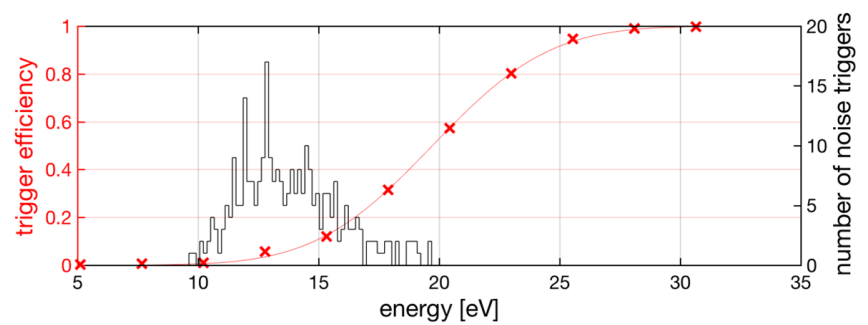

FIG. 8. Determination of the trigger threshold. Randomly chosen noise samples are superimposed with template pulses of different discrete energies (red crosses). The optimum trigger is applied to these samples yielding the energy-dependent trigger efficiency (left y axis). The data are fitted by an error function, giving an energy threshold of $E_{\mathrm{th}}=(19.7 \pm 0.1) \mathrm{eV}$ for $50 \%$ efficiency. The width $\sigma_{t h}=(3.82 \pm 0.15) \mathrm{eV}$ is in agreement with the variance of the baseline noise. The reconstructed energy of pure noise samples after filtering is shown in a histogram (black, right y axis).

the optimum filter output. The artificially added pulse clearly is seen above a given threshold (dotted line), while the random noise, which has a different pulse shape, is suppressed.

In the following we discuss how the energy threshold and the trigger efficiency can be determined in a direct way. Generally speaking, the threshold on the output of the optimum filter has to be chosen so as to be sensitive to the smallest possible energy depositions, while at the same time suppressing noise triggers sufficiently. Figure 8 (histogram, right axis) shows the filter output of a set of pure noise samples. In contrast to the determination of the baseline noise (see above), the pulse position (in time) is not fixed but the algorithm runs over the noise trace and returns the maximal filter output. This explains the positive average reconstructed energy. The bulk of the noise samples has a reconstructed energy between 10 and $15 \mathrm{eV}$ with a tail up to $\sim 19 \mathrm{eV}$. Most probably the latter is due to small pulses on the noise samples which cannot by identified by data-quality cuts selecting the noise samples. This effect is enhanced due to the exponentially increasing rate towards threshold in this calibration measurement. It is reasonable to set the trigger threshold just above this assumed noise population.

We choose a trigger threshold of $13.0 \mathrm{mV}$ and validate this choice by a study of the trigger efficiency as a function of energy. Onto the set of baseline samples, template pulses of various discrete pulse heights (from about 1 to $10 \cdot \sigma_{b}$ ) are added. The energy-dependent trigger efficiency is the fraction of the filtered artificial pulse samples which fall above the threshold. Figure 8 (left axis) shows the results of this procedure for the discrete pulse heights (crosses). The resulting curve can be nicely fitted by the function $p_{\text {trig }}(E)=0.5 \cdot\left(1+\operatorname{erf}\left[\left(E-E_{\text {th }}\right) /\left(\sqrt{2} \sigma_{\text {th }}\right)\right]\right.$, where erf is the Gaussian error function [11]. The validity of the threshold choice manifests itself as a vanishing trigger 
efficiency at low energies, corresponding to negligible noise triggers. Furthermore, the width of the error function is $\sigma_{\text {th }}=(3.83 \pm 0.15) \mathrm{eV}$, which is in good agreement with the baseline noise of $\sigma_{b}=(3.74 \pm 0.21) \mathrm{eV}$, demonstrating that the resolution of the detector at these energies is dominated by the baseline noise. The energy threshold (by definition at 50\% trigger efficiency) is found to be $E_{\text {th }}=(19.7 \pm 0.1$ (stat) $) \mathrm{eV}$, which corresponds to $5.27 \sigma_{b}$. In a setup with optimized noise conditions, the threshold can presumably be lowered to $4.5-5 \sigma_{b}$, a typical value reached in low-background underground environments [11]. Considering the systematic errors of the energy calibrations (see above), the energy threshold is $E_{\mathrm{th}}=(19.7 \pm 0.9) \mathrm{eV}$.

\section{Discussion of results}

The prototype detector shows the lowest energy threshold reported for macroscopic calorimeters, an improvement by 1 order of magnitude with respect to previous results [6]. It should be stressed that, since the device is a calorimeter, its calibration does not rely on quenching factors that arise when dealing with ionization or scintillation detectors. The detector has the same response for a given energy deposit, regardless of the interacting particle type and can approach energies below the fundamental nuclear-recoil reach of ionization detectors (e.g. $40 \mathrm{eV}$ for $\mathrm{Si}$ and $\mathrm{Ge}$ [18]). The threshold, shown as a blue circle in Fig. 2, is higher than predicted by the scaling law, indicated by the red stars. However, this is expected to improve in a low-noise, lowbackground environment. When operated in a low-noise underground setup, the energy threshold of the investigated $\mathrm{Al}_{2} \mathrm{O}_{3}$ detector is expected to improve by a factor of $1.5-3$ [6], to a value between 6 and $13 \mathrm{eV}$. Further improvements (e.g. optimizing the TES design) are foreseen to fully match the expectation of $4 \pm 1 \mathrm{eV}$.

The threshold is determined by comparing the measured noise with the amplitude of template pulses calibrated with events induced by the ${ }^{55} \mathrm{Fe}$ source. Thus the main assumption in arriving at our very low threshold is in the linear extrapolation of the pulse height-energy relation to low energies. Our threshold lies in a new energy range, never explored with this type of detector. Nevertheless, such low energies still correspond to the creation of very many of the $\mathcal{O}(1 \mathrm{meV})$ nonthermal phonons involved in the detection mechanism [8]. For energy depositions around or below $10 \mathrm{eV}$ there is the possibility of different nuclear-recoil mechanisms as one approaches lattice dislocation energies. It could be interesting for rare-event searches to attempt a direct calibration at lowest energies, as with recoils from neutron capture, nuclear isomeric transitions or electron capture.

\section{OUTLOOK FOR RARE-EVENT SEARCHES}

Due to the smallness of the calorimeters presented here, the technology permits new experiments in three aspects: (1) ultralow energy thresholds down to the $10 \mathrm{eV}$ regime, (2) encapsulation of the small calorimeters by cryogenic veto detectors and (3) ability to operate the detectors above ground in a high-rate environment. These features enable interesting possibilities involving the exploitation of the enhancement of the cross section by coherent scattering [19].

A new energy regime of nuclear recoils is accessible for the first time with this technology, which implies that a new range of DM particles can be probed. The prototype run described here can be used to set a new limit on the spin-independent DM particle-nucleon cross section $\sigma_{\mathrm{SI}}$ for masses below $m_{\mathrm{DM}}=500 \mathrm{MeV} / \mathrm{c}^{2}$ [20], extending the reach of direct DM search experiments down to $m_{\mathrm{DM}}=150 \mathrm{MeV} / \mathrm{c}^{2}$. Operated in a low-background setup gram-scale detectors will significantly improve in sensitivity. Assuming the present detector performance using $\mathrm{Al}_{2} \mathrm{O}_{3}$ and a background level of 10 counts/(kg keV day), which seems feasible even at a shallow site [3], an upper limit for $\sigma_{\mathrm{SI}}$ of $\sim 10^{-5} \mathrm{pb}\left(\right.$ at $500 \mathrm{MeV} / \mathrm{c}^{2}$ ) can be achieved with a moderate exposure of $1 \mathrm{~kg} /$ day. Improvements on the threshold, as predicted by the presented scaling law, might enable searches for $\mathrm{DM}$ masses in the $10 \mathrm{MeV}$ regime.

The rate of solar neutrinos scattering coherently on nulcei in $\mathrm{CaWO}_{4}$ exceeds the present intrinsic background level at recoil energies below $\sim 7 \mathrm{eV}$, as shown in Fig. 1. In this energy regime, the spectrum is dominated by scatters from $\mathrm{Be}^{7}$ and pp neutrinos of the solar cycle. An array of gram-scale detectors with a total target mass of $\mathrm{O}(1 \mathrm{~kg})$ would allow flavor-independent precision measurements of the solar neutrino flux and enable new solar physics [21].

The technology presented here shows promise for a rapid detection of CNNS and opens a window to study many interesting physics scenarios beyond the standard model [22]. Lowering the energy threshold from, e.g. 300 to $20 \mathrm{eV}$ boosts the expected count rate by about 2 orders of magnitude (Fig. 1). Operating an array of such detectors with a total mass of $10 \mathrm{~g}$ at a distance of $40 \mathrm{~m}$ from a $\mathrm{GW}$-scale nuclear power reactor yields a count rate of $\sim 10^{3}$ counts / $\left(\mathrm{kg} \mathrm{keV}\right.$ day), which is a factor of $10-10^{3}$ above expected backgrounds (see Fig. 1 and [15]). A $5 \sigma$ discovery of CNNS is then expected within $\lesssim 2$ weeks of measuring time, and precision measurements of the cross section are in reach. Such a small-scale experiment can be realized with a commercial cryostat, standard lab electronics and a (presumably) compact shielding, which results in a moderate cost estimate. With this technology, real-time monitoring of nuclear reactors for nonproliferation and accident control is in reach. 
[1] L. Stodolsky, Phys. Today 44, No. 8, 24 (1991).

[2] G. Angloher et al., Eur. Phys. J. C 76, 25 (2016).

[3] H. Gastrich, C. Gößling, R. Klingenberg, K. Kröninger, T. Neddermann, C. Nitsch, T. Quante, and K. Zuber, Appl. Radiat. Isot. 112, 165 (2016).

[4] D. S. Akerib et al., Phys. Rev. D 82, 122004 (2010).

[5] R. Strauss et al., J. Cosmol. Astropart. Phys. 06 (2015) 030.

[6] R. Strauss et al., Nucl. Instrum. Methods Phys. Res., Sect. A 845, 414 (2017).

[7] G. Angloher et al., Eur. Phys. J. C 72, 1971 (2012).

[8] F. Pröbst, M. Frank, S. Cooper, P. Colling, D. Dummer, P. Ferger, G. Forster, A. Nucciotti, W. Seidel, and L. Stodolsky, J. Low Temp. Phys. 100, 69 (1995).

[9] S. H. Moseley, J. C. Mather, and D. McCammon, J. Appl. Phys. 56, 1257 (1984).

[10] J. A. Formaggio, E. Figueroa-Feliciano, and A. J. Anderson, Phys. Rev. D 85, 013009 (2012).

[11] G. Angloher et al., Eur. Phys. J. C 74, 3184 (2014).

[12] O. Meier et al., Nucl. Instrum. Methods Phys. Res., Sect. A 444, 350 (2000).
[13] G. Angloher, M. Bauer, I. Bavykina, A. Bento, A. Brown, C. Bucci, C. Ciemniak, C. Coppi, G. Deuter, and F. von Feilitzsch, Astropart. Phys. 31, 270 (2009).

[14] M. Sisti et al., Nucl. Instrum. Methods Phys. Res., Sect. A 466, 499 (2001).

[15] R. Strauss et al., arXiv:1704.04320 [Eur. Phys. J. C (to be published)].

[16] E. Gatti and P. F. Manfredi, Riv. Nuovo Cimento 9, 1 (1986).

[17] G. Piperno, S. Pirro, and M. Vignati, J. Instrum. 6, P10005 (2011).

[18] R. Agnese et al., Phys. Rev. D 95, 082002 (2017).

[19] A. Drukier and L. Stodolsky, Phys. Rev. D 30, 2295 (1984).

[20] A. Angloher et al. (CRESST Collaboration), arXiv: 1707.06749.

[21] D. G. Cerdeño, M. Fairbairn, T. Jubb, P. A. N. Machado, A. C. Vincent, and C. Bœhm, J. High Energy Phys. 05 (2016) 118.

[22] M. Lindner, W. Rodejohann, and X.-J. Xu, J. High Energy Phys. 03 (2017) 097. 